\title{
Low-Density Parity-Check Codes for 40-Gb/s Optical Transmission Systems
}

\author{
Ivan B. Djordjevic, Member, IEEE, Sundararajan Sankaranarayanan, Shashi Kiran Chilappagari, \\ and Bane Vasic, Senior Member, IEEE
}

\begin{abstract}
In this paper, we compare performance of three classes of forward error correction schemes for $40-\mathrm{Gb} / \mathrm{s}$ optical transmission systems. The first class is based on the concatenation of Reed-Solomon codes and this is employed in the state-of-theart fiber-optics communication systems. The second class is the turbo product codes with Bose-Chaudhuri-Hocquenghen component codes. The application of these codes in optical communication systems was extensively studied by Sab and Lemarie, and Mizuochi et al. The third class is the low-density parity-check (LDPC) codes that have attracted much attention over the past decade. We present enhanced decoding algorithms for Turbo product codes and LDPC codes that use probability density function of output sequences instead of calculating initial likelihood ratios assuming (inaccurate) Gaussian or chi-square approximation. The analysis in this paper shows that the LDPC codes perform better than the other codes in the waterfall region at bit error rates as low as $10^{-9}$. We also presented error floors results obtained by analyzing decoding failures of hard-decision iterative decoders.
\end{abstract}

Index Terms-Error floor, low-density parity-check (LDPC) codes, optical communications, turbo product codes.

\section{INTRODUCTION}

$\mathbf{T}$ HE state-of-the-art fiber-optics communication systems standardized by the ITU employ concatenated BoseChaudhuri-Hocquenghen/Reed-Solomon (BCH/RS) codes [1], [2]. In recent years, iteratively decodable codes, like turbo codes [3]-[6] and low-density parity-check (LDPC) codes [7]-[12], have generated significant research attention. In [4], Sab and Lemarie proposed a forward error correction (FEC) scheme based on block turbo code for long-haul DWDM optical transmission systems. In recent papers [7]-[11], we have shown that iteratively decodable LDPC codes outperform turbo product codes. The decoder complexity of these codes is comparable (or lower) to that of turbo product codes and significantly lower than that of serial/parallel concatenated turbo codes. For reasons mentioned above, LDPC code is a viable and attractive choice for the FEC scheme of a 40-Gb/s optical transmission system.

Although iterative decoding schemes have been intensively studied recently, the following list of important issues has not been addressed in the existing literature: 1) a systematic com-

Manuscript received August 15, 2005; revised February 27, 2006. This work was supported by the National Science Foundation under Grant ITR 0325979.

I. B. Djordjevic, S. K. Chilappagari, and B. Vasic are with the Department of Electrical and Computer Engineering, University of Arizona, Tucson, AZ 85721 USA (e-mail: ivan@ece.arizona.edu; shashic@ece.arizona.edu; vasic@ ece.arizona.edu).

S. Sankaranarayanan was with the Department of Electrical and Computer Engineering, University of Arizona, Tucson, AZ 85721 USA. He is now with Seagate Technologies, Pittsburgh, PA 15222 USA (e-mail: ssankara@seagate. com).

Digital Object Identifier 10.1109/JSTQE.2006.876182 parison of different classes of FEC in a realistic simulation environment that captures all impairing effects in a long-haul transmission system; 2) the initial likelihoods fed to an iterative decoder are typically calculated assuming Gaussian or chi-square distributions of received samples without validating these distributions; and 3) understanding the phenomenon of error floors in performance curves of iteratively decoded LDPC codes. The aim of this paper is to address the above-mentioned issues.

We compare the bit error rate (BER) performance of three different classes of FEC schemes: 1) concatenation of two RS codes; 2) turbo product codes; and 3) structured LDPC codes. The BER performance in the waterfall region is computed using a simulation model that takes into account the signal-amplified spontaneous emission (ASE) noise interaction during transmission and other important transmission impairments. The LDPC codes of interest in this paper are finite geometry codes, block-circulant codes, lattice codes of girth 8 , and nonbinary LDPC codes.

In the existing literature, the BER performance of both turbo product codes [5] and LDPC codes [12] is assessed assuming that noise is white and zero mean Gaussian distributed. However, a decoder built on such an assumption degrades the BER performance, as shown later in the text. Hence, we propose to estimate the probability density functions of channel output sequences and use those estimates in assigning initial likelihoods.

The BER or frame error rate (FER) performance in the high signal-to-noise ratio region (error floor region) of iterative decoders is still an open problem in coding theory, although some progress has been made recently [27], [28], [30], [31]. Optical communications systems operate at extremely low BERs (or FERs), which makes estimating error floor by Monte-Carlo simulations virtually impossible. In this paper, we present FER of finite geometry codes computed semianalytically. With an aim to decouple precision issues of the decoder implementation from the analysis, we consider iterative hard-decision messagepassing algorithm, commonly referred to as Gallager B algorithm [29].

This paper is organized as follows. The calculation of initial bit reliabilities based on estimated probability density functions is described in Section II. Also, Section II provides a description of the transmission system and dispersion map under study. In Section III, we compare the performance of structured LDPC codes with that of the turbo product code and a concatenation of RS codes. In Section IV, we present results on analytical and semianalytical computation of block error rate of finite geometry codes. Finally, Section V concludes the paper. For 
completeness of the paper, a brief description of LDPC and turbo product decoding algorithms is provided in Appendixes A and $\mathrm{B}$, respectively.

\section{CAlculation OF INITIAl Log-LiKElihood Ratios}

The performance of iterative decodable codes is commonly assessed using one of the following channel models: Gaussian model [2], chi-square model [32], and colored Gaussian model [33]. Recently, the performance of different classes of LDPC codes was assessed using an advanced simulator that takes into account all major transmission impairments, such as Kerr nonlinearities (both interchannel and intrachannel nonlinearities), stimulated Raman scattering, first and second order group-velocity dispersions, ASE noise, filtering effects (both optical and electrical), intersymbol interference, and linear crosstalk effects (see [7]-[11]). In order to speed up simulations, a long encoded sequence was passed through the transmission system once, and equivalent ASE noise was added at the receiver end. However, this approach ignores the effects of ASE noise and signal interaction during transmission. The above effects, especially intrachhannel nonlinearites, such as intrachannel four-wave mixing (IFWM), intrachannel crossphase modulation (IXPM), and self-phase modulation (SPM) are significant source of errors at data speeds of $40 \mathrm{~Gb} / \mathrm{s}$ or above [34].

In this section, we describe a method of calculating the initial log-likelihood ratios (LLRs), used as inputs in an iterative decoder (turbo product or LDPC decoder). To determine the LLRs, we have to determine the probability density functions (PDF) for mark- and space-state bits, using the simulator. To obtain a good estimate of the PDF, an accurate channel model is crucial. In Section II-A, we explain the channel model that takes into account the transmission impairments mentioned above. Section II-A also gives a verification of the model. The calculation of initial likelihoods is described in Section II-B.

\section{A. Simulator Description and Verification}

The system of interest in this paper is a wavelength division multiplexing (WDM) system. The continuous-wave laser signals at different wavelengths are modulated using independently encoded electrical streams and a Mach-Zehnder (MZ) modulator, WDM multiplexed and transmitted over the same fiber. The return-to-zero on-off keying (RZ-OOK) and carriersuppressed RZ (CSRZ) OOK modulators, shown in Fig. 1(b), are composed of a laser diode, two MZ intensity modulators (the first serving as a modulator, the second as an NRZ-RZ converter), a PRBS generator, and an encoder. RZ/CSRZ receiver configuration is given in Fig. 1(c). It is composed of a WDM demultiplexer (modeled as an optical filter), a PIN photodiode, an electrical filter, and a sampler followed by a decoder. An Erbium-doped fiber amplifier (EDFA) is used as a preamplifier. RZ-DPSK transmitter and receiver configurations are given in Fig. 1(d) and (e), respectively. For more details on RZ-DPSK, reader may refer [25]. EDFAs and dispersion compensating fibers (DCF or $D_{-}$fiber) are deployed periodically to

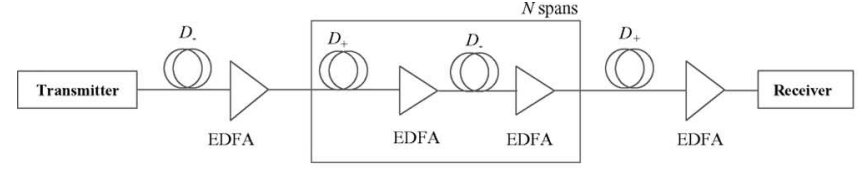

(a)

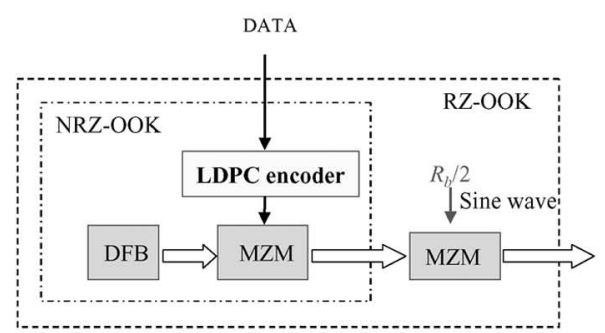

(b)

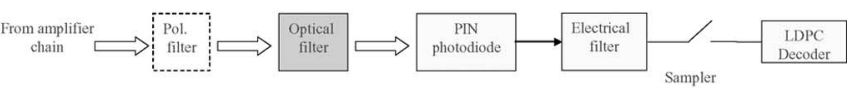

(c)

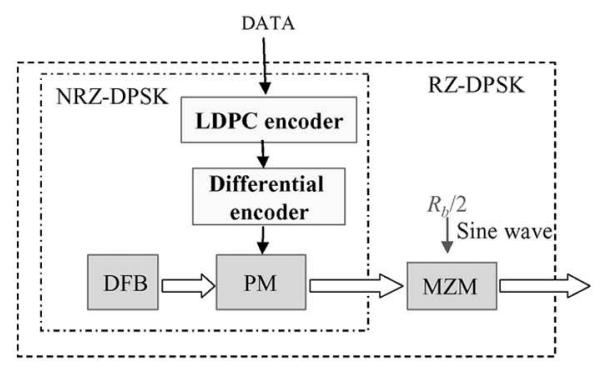

(d)

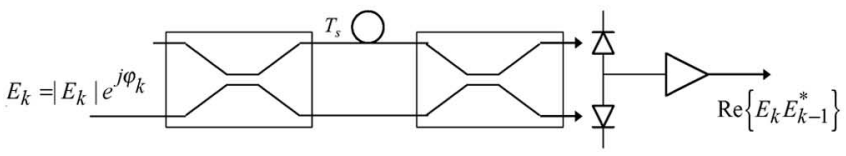

(e)

Fig. 1. (a) Dispersion map under study. (b) RZ/CSRZ transmitter configuration. (c) RZ/CSRZ receiver configuration. (d) RZ-DPSK transmitter configuration. (e) RZ-DPSK receiver configuration. DFB: Distributed-feedback laser; MZM: Mach-Zehnder modulator; PM: Phase modulator; $T_{\mathrm{s}}$ : Symbol duration.

compensate the loss and accumulated dispersion of the standard single mode fiber (SMF or $\mathrm{D}_{+}$fiber). The propagation of a signal through the transmission media is modeled by a nonlinear Schrödinger equation (NLSE) [38]

$$
\begin{aligned}
\frac{\partial A}{\partial z}= & -\frac{\alpha}{2} A-\frac{i}{2} \beta_{2} \frac{\partial^{2} A}{\partial T^{2}}+\frac{\beta_{3}}{6} \frac{\partial^{3} A}{\partial T^{3}} \\
& +i \gamma\left(|A|^{2}-T_{R} \frac{\partial|A|^{2}}{\partial T}\right) A
\end{aligned}
$$

where $z$ is the propagation distance along the fiber, relative time $T=t-z / v_{g}$ gives a frame of reference moving at the group velocity $v_{\mathrm{g}}, A(z, T)$ is the complex field amplitude of the pulse, $\alpha$ is the attenuation coefficient of the fiber, $\beta_{2}$ is the group velocity dispersion (GVD) coefficient, $\beta_{3}$ is the secondorder GVD, and $\gamma$ is the nonlinearity coefficient giving rise to Kerr effect nonlinearities. Such nonlinearities include self-phase modulation, intrachannel four-wave mixing, intrachannel crossphase modulation, cross-phase modulation (XPM), four-wave 


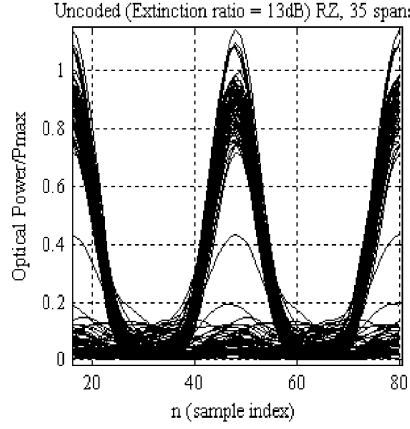

(a)

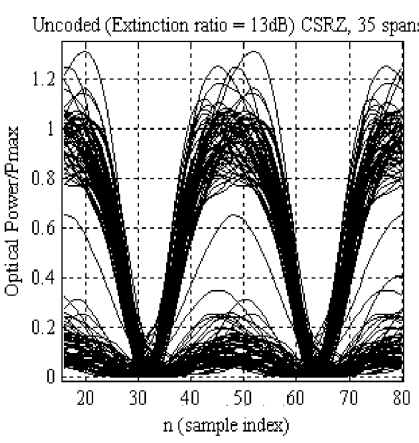

(b)
Fig. 2. Eye diagrams after 35 spans of dispersion map in Fig. 1 for extinction ratio of $13 \mathrm{~dB}$. (a) RZ. (b) CSRZ.

mixing (FWM); with $T_{\mathrm{R}}$ is denoted as the Raman coefficient describing the stimulated Raman scattering (SRS). The NLSE was solved using the split-step Fourier method [38].

Dispersion map under study is shown in Fig. 1(a). The parameters of $\mathrm{D}_{+}$and $\mathrm{D}_{-}$fibers may be found in [7], the span length is set to $L=48 \mathrm{~km}$ and each span consists of $2 L / 3 \mathrm{~km}$ of $\mathrm{D}_{+}$fiber followed by $L / 3 \mathrm{~km}$ of $\mathrm{D}_{-}$fiber. Precompensation of $-320 \mathrm{ps} / \mathrm{nm}$ and corresponding postcompensation are also applied. RZ-OOK modulation format of duty cycle 33\%, CSRZ-OOK, and RZ-DPSK are observed. EDFA noise figure is set to $6 \mathrm{~dB}$, the bandwidth of optical filter is set to $2 R_{\mathrm{b}}$, and the bandwidth of electrical filter is set to $0.65 R_{\mathrm{b}}$, with $R_{\mathrm{b}}$ being the bit rate $(40 \mathrm{~Gb} / \mathrm{s})$. In a WDM environment (Fig. 5), the channel spacing is set to $100 \mathrm{GHz}$, and the six neighboring channels influencing the observed channel at $1552.524 \mathrm{~nm}$ are taken into consideration. The Raman coefficient $T_{\mathrm{R}}$ is set to a typical value $3.0 \times 10^{-15}$ [38].

As demonstrated in our recent publications [21], [22], the simulator used to estimate the probability density functions for mark- and state-bits, described above, gives an excellent agreement with commercially available VPI transmissionMaker WDM version 5.5. For example, in Fig. 2, the eye diagrams after 35 spans of RZ and CSRZ at $40 \mathrm{~Gb} / \mathrm{s}$ (with the extinction ratio of $13 \mathrm{~dB}$ ), for dispersion map in Fig. 1, are shown as an illustration. The amplitude jitter and ghost pulse effects due to IFWM and the timing jitter due to IXPM may easily be identified, suggesting that the simulator is able to catch all important nonlinear effects. In Section II-B, the procedure to determine the initial LLRs is described.

\section{B. Log-likelihood Ratios and Extrinsic Information}

Turbo product codes and LDPC codes are soft-decoded iteratively, using the Chase II algorithm and sum-product algorithm (SPA), respectively. To overcome the inaccuracy in Gaussian approximation of initial log-likelihood ratios, the probability density functions of received samples are calculated from simulation.

Let $u_{j}$ be the $j$ th bit in a codeword $\boldsymbol{u}$ and $r_{j}$ be the corresponding received sample. The transition probability $p\left(r_{j} \mid s\right)$ is estimated from simulator by modeling the channel as finite state machine ( $s$ denotes the state of the channel). It is assumed

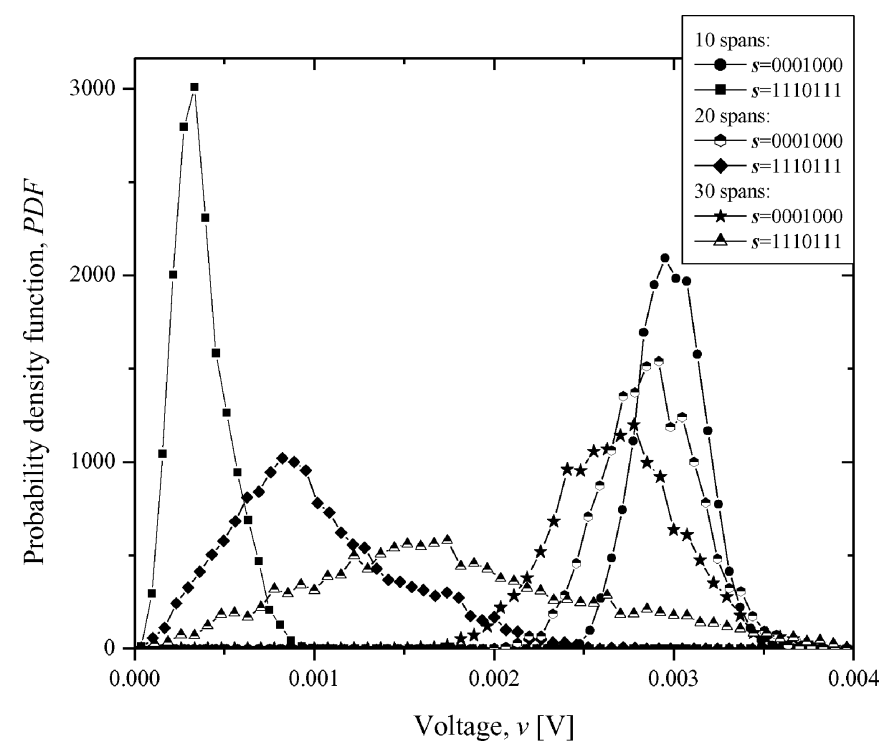

Fig. 3. Probability density functions estimated from simulator for RZ-OOK.

that $m$ previous and $m$ next bits influence the observed bit $u_{j}$, and the state $s=\left(u_{j-m}, u_{j-m+1}, \ldots, u_{j}, u_{j+1}, \ldots, u_{j+m}\right)$ is determined by a sequence of $2 m+1$ input bits $u_{i} \in\{0,1\}$. Fig. 3 shows the conditional PDFs obtained for the dispersion map described in Fig. 1(a) with launched power of $0 \mathrm{dBm}$, for memory $2 m+1=7$. A span length is set to $120 \mathrm{~km}$ and precompensation of $-800 \mathrm{ps} / \mathrm{nm}$ is assumed. As expected, by increasing the number of spans, the ghost pulse at the central bit position for the state $s=1110111$ grows, hence shifting the mean of the PDF to the right. On the other hand, the mean of PDF for an isolated one (in state $s=0001000$ ) shifts to the left as the number of spans increases, suggesting that the assumed memory 7 is not sufficient enough to capture the effect of intersymbol interference. To estimate the PDFs, the region of all possible samples is quantized in 64 bins, and the number of occurrences of samples in a given bin is counted and normalized with total number of samples. From Fig. 3, it is evident that different states have different PDFs; therefore, to determine the PDF of mark-/space-state bit, the PDFs for all states in which the middle mark/state bit is involved are to be averaged

$$
P\left(r_{j} \mid u_{j}=0,1\right)=\sum_{\mathbf{s}: u_{j}=0,1} P\left(r_{j} \mid s\right) P(s) .
$$

Although the method has limited accuracy, it serves very well the purpose. It was found that an increase in number of bins from 64 to 128 and an increase in memory from 11 to 13 did not show a significant improvement of the BER.

By determining the transition probabilities $P\left(r_{j} \mid u_{j}\right)$ and assuming that a priori probabilities $P\left(u_{j}\right)$ are known, Bayes theorem gives the conditional probability of the observed bit $u_{j}$, given the received sample $r_{j}$ as

$$
\begin{aligned}
& P\left(u_{j} \mid r_{j}\right)= \\
& \frac{P\left(r_{j} \mid u_{j}\right) P\left(u_{j}\right)}{p\left(r_{j} \mid u_{j}=0\right) P\left(u_{j}=0\right)+P\left(r_{j} \mid u_{j}=1\right) P\left(u_{j}=1\right)} .
\end{aligned}
$$


Finally, the initial likelihood of bits can be calculated as

$$
L\left(u_{j}\right)=\log \frac{P\left(u_{j}=0 \mid r_{j}\right)}{P\left(u_{j}=1 \mid r_{j}\right)} .
$$

These initial LLRs are fed to an iterative decoder or used to determine hard decisions for a RS code. The LDPC decoding algorithm operates by passing the messages on bipartite graphs associated with a parity-check matrix of a code and hence, it is also known as the message-passing (MP) algorithm or sumproduct algorithm [16] and is briefly explained in Appendix A.

Decoding of the turbo product codes is based on an efficient implementation of Chase II algorithm [17], [18], as explained in Appendix B. The initial likelihoods of candidate codewords $c_{i}$ are calculated by

$$
\begin{aligned}
\Lambda\left[\boldsymbol{c}_{i}\right. & \left.=\left(\boldsymbol{c}_{i}(1) \boldsymbol{c}_{i}(2) \ldots \boldsymbol{c}_{i}(n)\right)\right] \\
& =\sum_{j=1}^{n} \log \left[\frac{\exp \left[\left(1-\boldsymbol{c}_{i}(j)\right) L\left(\boldsymbol{c}_{i}(j)\right)\right]}{1+\exp \left[L\left(\boldsymbol{c}_{i}(j)\right)\right]}\right]
\end{aligned}
$$

where the bit likelihoods $L\left[\mathbf{c}_{i}(j)\right]$ are determined from (4). We use $\boldsymbol{c}_{i}(j)$ to denote the $j$ th component of the $i$ th candidate codeword $c_{i}$. The initial bit reliabilities for turbo decoder are calculated by

$$
\lambda\left(u_{j}\right)=\log \left[\frac{\sum_{\boldsymbol{c}_{i}(j)=0} \Lambda\left(\boldsymbol{c}_{i}\right)}{\sum_{\boldsymbol{c}_{i}(j)=1} \Lambda\left(\boldsymbol{c}_{i}\right)}\right] .
$$

The extrinsic reliabilities for next decoding stage are calculated by

$$
L_{\text {ext }}\left(u_{j}\right)=\lambda\left(u_{j}\right)-L\left(u_{j}\right) .
$$

In calculation of the candidate codeword LLRs and extrinsic reliabilities no approximations are made. So, there is no need to introduce any correction factor. There are two main differences compared to previously proposed algorithms [4]-[6]: 1) the initial likelihoods are estimated from the channel by determination of PDFs, while in [4]-[6] the initial likelihoods are determined assuming Gaussian distribution and 2) all possible candidate codewords LLRs are used in calculation of extrinsic reliabilities.

The hard decisions for RS concatenation scheme are determined from (4) by

$$
\hat{u}_{j}=\left\{\begin{array}{ll}
1, & \text { if } L\left(u_{j}\right)<0 \\
0, & \text { otherwise }
\end{array} .\right.
$$

\section{COMPARISON OF STRUCTURED LDPC CODES AND TURBo PRODUCT CODES}

Although random like long LDPC codes (e.g., [12]) are able to approach the Shannon's limit, the complexity of LDPC encoders and decoders can be considerably reduced by allowing cyclic or quasi-cyclic structures in the parity-check matrices of the codes. Especially, these symmetries are critical to enable high-speed FEC architectures for optical communications. The cyclic or quasi-cyclic structures of these codes support simple encoders, realized using shift registers and modulo- 2 adders, and lowcomplex iterative decoders. The decoder implementation issues are beyond the scope of this paper, but it is a topic that attracts significant research attention. Briefly, the complexity of SPA for nonbinary codes is higher [14] compared to that of binary LDPC codes [16]. However, the highly regular structure of parity-check matrix of block-circulant codes proposed by authors in [15] facilitates the implementation. Moreover, the basic operations in lower Galois field are not difficult to implement (see [35]), and recently several efficient iterative decoding algorithms for nonbinary LDPC codes have been proposed [14], [36], [37]. It should be also noted that the complexity of Chase II algorithm explained in Appendix B is lower compared to the complexity of SPA [16]. However, during the decoding process, turbo product decoder (for $\mathrm{BCH}(128,113) \times \mathrm{BCH}(256,239))$ has to employ 239 Chase II blocks operating in parallel, increasing therefore the decoding delay and the circuit size. Moreover, the turbo product codes require the use of interleaver. For more details about the implementation of SPA reader may refer [26].

In [9], we presented a construction method based on the incidence of points and lines of a finite projective plane, and we extended the above construction method to include affine planes, and some secondary structures in projective planes like ovals, and unitals. In [7]-[11], we proposed several LDPC designs based on integer lattice, mutually orthogonal Latin squares and rectangles, and block-circulant parity check matrices. These codes have shown encouraging performance with coding gains greater than that of the best turbo codes [4]-[6] proposed for optical communication systems.

In the calculation of BER performance for different classes of codes for 40-Gb/s transmission, shown in Fig. 4(b), an encoded sequence of length $2^{15}$ is transmitted many times over the whole transmission system for different ASE noise realizations. The number of spans is changed from 20 to 160 depending on the modulation format and error control coding scheme being employed. In calculation of initial likelihoods, the PDFs determined from simulation are employed, as explained in Section II. Such an approach gives accurate BER estimates.

In Fig. 4, the BER performance of block-circulant codes and projective geometry codes of girth 6 and lattice codes of girth 8 are compared against the turbo product codes from [6] and RS concatenation scheme from [2]. The results for an AWGN channel are shown in Fig. 4(a), and results for dispersion map from Fig. 1(a) and single channel transmission are shown in Fig. 4(b).

On the AWGN channel, the turbo product code $\mathrm{BCH}(128,113) \times \mathrm{BCH}(256,239)$ (with the number of least reliable positions in Chase II algorithm set to $p=5$, and the number of iterations set to 5) provides a 2.45-dB improvement in (net effective) coding gain at BER of $7.573 \times 10^{-8}$ over $\operatorname{RS}(255,239)+\operatorname{RS}(255,223)$ concatenation scheme. Blockcirculant $(8176,6761,0.821)$-LDPC code provides a $2.72-\mathrm{dB}$ improvement over RS concatenation scheme and a $0.27-\mathrm{dB}$ improvement over turbo product code. Lattice code of girth 8, (8547, 6922, 0.81)-LDPC code, provides 2.94-dB improvement over RS concatenation scheme and $0.49 \mathrm{~dB}$ over turbo product code. $(8547,6922,0.81)$-LDPC code over GF(8) provides a 3.14-dB improvement over RS concatenated code, and a 0.69-dB improvement over turbo product code. Note that RS 


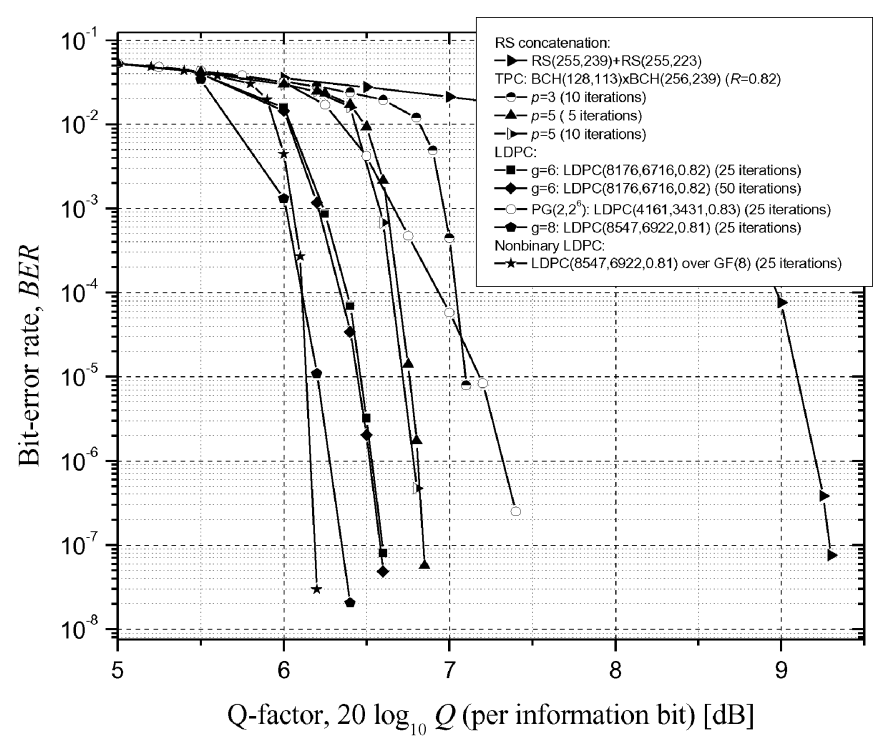

(a)

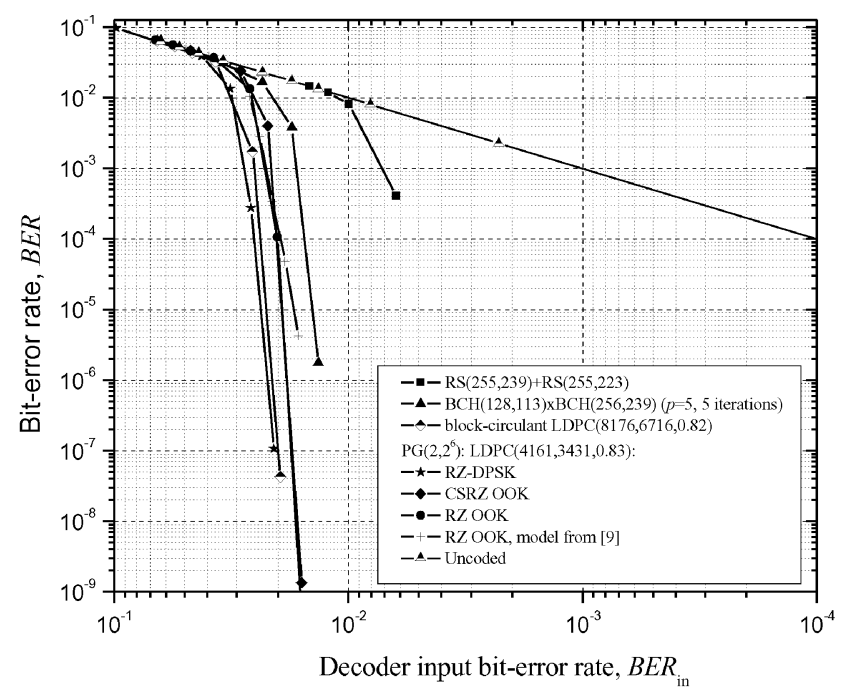

(b)

Fig. 4. Binary LDPC codes against turbo product codes and RS concatenation coding scheme for (a) AWGN channel model and (b) dispersion map from Fig. 1 for single channel transmission at $40 \mathrm{~Gb} / \mathrm{s}$.

concatenated code, turbo product code, and LDPC codes are carefully designed to have similar code rates. However, the turbo product code is much longer than that of LDPC codes. The codeword length of RS concatenation scheme is significantly longer (15 and 63 times, respectively) than the codeword length of both turbo product codes and LDPC codes.

Note that the LDPC codes do not require the use of interleaver, while for both RS concatenation scheme and turbo product code simple block interleaver is employed, as shown in Appendix B. Note that the interleavers in [2] and [6] are different, and the improved version of Chase II algorithm (described in Appendix B) is different from that in [6].

On AWGN channel, turbo product code outperforms significantly shorter PG $\left(2,2^{6}\right)$ based $(4161,3431,0.825)$-LDPC code. However, as shown in Fig. 4(b), on fiber optics channel model (from Fig. 1), PG $\left(2,2^{6}\right)$ code for RZ modulation format sig-

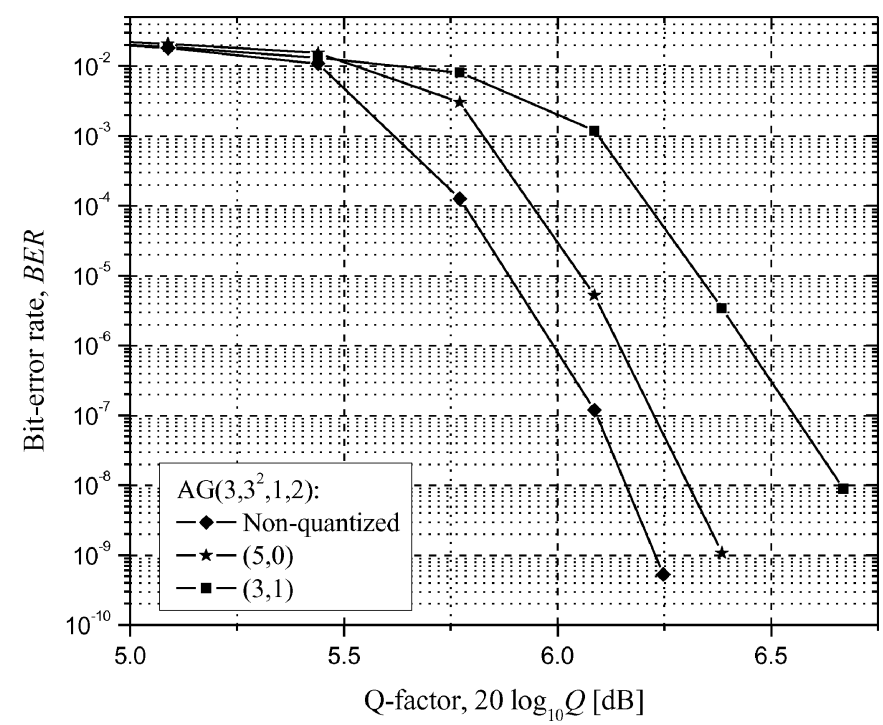

Fig. 5. Performance loss due to fixed-point representation of LLRs.

nificantly outperforms the turbo product code, suggesting that LDPC codes are much more powerful in correcting error bursts due to Kerr nonlinearities, such as IFWM. RZ-DPSK is superior to both RZ-OOK and CSRZ-OOK, while RZ-OOK and CSRZOOK are comparable for $\mathrm{PG}\left(2,2^{6}\right)$ based code. From Fig. 4(b) it is evident that BER prediction in which the ASE noise-signal interaction during transmission is ignored (the cross curve) is underestimated. Note also that the same BER for CSRZ is achieved for larger number of spans compared to RZ.

A double precision for LLRs is used to obtain the curves in Fig. 4. However, in practice, hardware implementations of the SPA require quantizing the LLRs. A fixed-point (FP) representation of a real-valued LLR $\lambda$ is an integer $\lambda_{\mathrm{z}}$ with an $n_{\mathrm{b}}$-bit precision [20]. Out of the $n_{\mathrm{b}}$ bits, $d_{\mathrm{b}}$ bits are used to represent the integer part (including the sign) of $\lambda$ and $p_{\mathrm{b}}$ bits are used to represent the decimal part of $\lambda$. The range of $\lambda_{\mathrm{z}}$ is defined by $\left(d_{\mathrm{b}}, p_{\mathrm{b}}\right)$, where $n_{\mathrm{b}}=p_{\mathrm{b}}+d_{\mathrm{b}}$. The FP representation of $\lambda$ is obtained as follows:

$$
\lambda_{\mathrm{z}}=\left\{\begin{array}{l}
\min \left(\left\lfloor 2^{p_{b}} \lambda+0.5\right\rfloor, 2^{n_{b}-1}-1\right) \\
\max \left(\left\lfloor 2^{p_{b}} \lambda+0.5\right\rfloor,-2^{n_{b}-1}\right) .
\end{array}\right.
$$

Hence, the range of $\lambda_{\mathrm{z}}$ is $\left[2^{n_{b}-1}-1,-2^{n_{b}-1}\right]$ and we refer to $\lambda_{\mathrm{z}}$ as $\left(d_{\mathrm{b}}, p_{\mathrm{b}}\right)$-quantized.

In the decoding stage, the intrinsic information obtained from the channel observations is $\left(d_{\mathrm{b}}, p_{\mathrm{b}}\right)$-quantized and fed to the FP iterative decoder. The result of any operation performed within the decoder is $\left(d_{\mathrm{b}}, p_{\mathrm{b}}\right)$-quantized. We observe from Fig. 5 that the performance loss due to 5-bit quantization is within $0.2 \mathrm{~dB}$ at BER of $10^{-9}$. Simulations are performed in WDM environment, as described in Section II-A. Thus, we have shown that carefully designed LDPC codes are able to outperform significantly both turbo product codes and RS concatenation schemes of comparable rates. Also, quantized implementation of the LDPC decoding algorithm results in negligible performance loss.

The $Q$-factor is calculated using the expression

$$
Q=\sqrt{2} \operatorname{erfc}^{-1}\left(2 \cdot P_{\mathrm{e}, \mathrm{unc}}\right)
$$

where $P_{\mathrm{e}, \mathrm{unc}}$ denotes the BER of uncoded signal. 


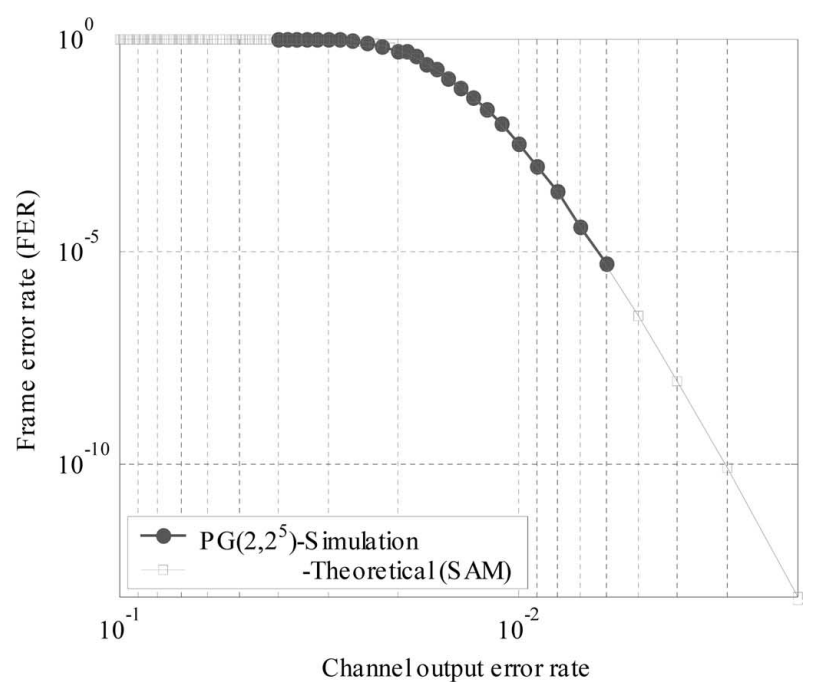

(a)

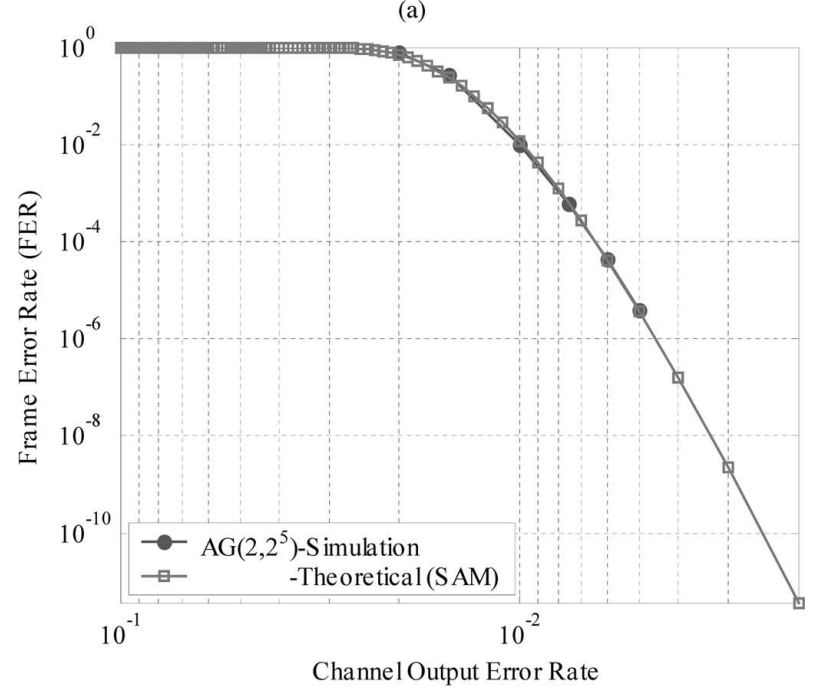

(b)

Fig. 6. Semianalytic method (SAM) for FER analysis of hard-decision decoding of (a) projective geometry codes and (b) affine geometry codes.

\section{SEMiAnAlytic Estimation OF FER}

In [27], Richardson proposed a semianalytical method to compute error floor in frame error rate (FER) curves of LDPC codes on AWGN channel. A decoding failure of the SPA was characterized using a combinatorial object called a trapping set. The support of a trapping set of a code is a fixed point of the decoder. At high SNRs, minimal (in terms of cardinality) trapping sets of a code contribute to its error floor. This contribution is dependent on the size of a minimal trapping set, the number of minimal trapping sets, and the decoding algorithm. This means that the error floor of a code varies with the precision of likelihood messages used for decoding. Hence, with an aim to understand the impact of code structures on the FER, we investigated trapping sets of LDPC codes decoded using the Gallager B algorithm (iterative hard-decision message-passing decoder) [29]. In this paper, we will restrict our analysis to codes from projective and affine planes.
The size of the minimal trapping set of codes from projective and affine planes (decoded using the Gallager B algorithm) is $\left\lceil\frac{c}{2}\right\rceil+1$, where $c$ is the column weight of a code. It can be shown that Gallager B algorithm is guaranteed to correct all error patterns of length up to $t$, where $t=\left\lfloor\frac{d-1}{2}\right\rfloor$ and $d$ is the minimum distance of the code. Another major cause for a decoding failure is characterized using an object called the propagating set. The support of an initial error pattern is a propagating set if it results in error propagation during the decoding process. A significant contributor to errors in decoding of codes from projective and affine plane is the propagating set. Using a semianalytical method, we have estimated the FER performance of these codes. These results are presented in Fig. 6.

Fig. 6 demonstrates that the theoretically estimated performance agrees with that computed from simulations, and there is no error floor in the region of interest for optical communications. For more information on error floor of LDPC codes, reader may refer to [30], [31].

\section{CONCLUSION}

The BER performance of structured LDPC codes are compared versus turbo product codes and RS concatenation scheme for initial bit reliabilities calculated from probability density functions determined from simulation. Different classes of LDPC codes outperform relatively longer turbo product codes of comparable rate. The performance loss due to quantization of log-likelihood ratios is found to be negligible. Finite geometry codes do not exhibit error floor in the region of interest for optical transmission.

\section{APPENDIX A \\ SPA OVERVIEW}

The SPA maybe described as follows. First, a priori information of the bit at position $j, \mu_{j}^{(0)}$, is calculated using (4), and messages passed from variable node $j$ to check node $c$ in the bipartite graph $\lambda_{j, c}^{(0)}$ are initialized to $\mu_{j}^{(0)}$. In the $i$ th iteration, we update the messages to be passed from check node $c$ to bit node $j, \Lambda_{c, j}^{(i)}$, as

$$
\Lambda_{c, j}^{(i)}=-2 \tanh ^{-1}\left[\prod_{w \neq j} \tanh \left(\lambda_{w, c}^{(i-1)} / 2\right)\right]
$$

and messages to be passed from bit node $j$ to check node $c, \lambda_{j, c}^{(i)}$, according to

$$
\lambda_{j, c}^{(i)}=\mu_{j}^{(0)}+\sum_{d \neq c} \Lambda_{d, j}^{(i)} .
$$

The last step in iteration $i$ is to compute updated loglikelihood ratios $\mu_{j}^{(i)}$ according to

$$
\mu_{j}^{(i)}=\mu_{j}^{(0)}+\sum_{c} \Lambda_{c, j}^{(i)} .
$$




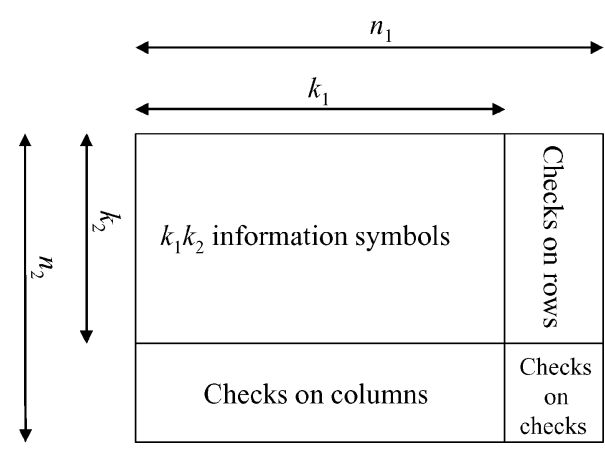

Fig. 7. Product code codeword structure.

For each bit $j$, the estimation is made according to

$$
\hat{u}_{j}= \begin{cases}1, & \text { if } \mu_{j}^{(i)}<0 \\ 0, & \text { otherwise. }\end{cases}
$$

Procedure halts when a valid codeword is generated or a maximum number of iteration has been reached.

The efficient realization of SPA proposed in [16] is employed in simulations, which allows additional $\sim 0.5$ - $\mathrm{dB}$ improvement in coding gain compared to the min-sum approximation of SPA implemented in [9].

\section{APPENDIX B}

\section{TuRbo PRODUCT Codes}

A product code [3]-[6] is an $\left(n_{1} n_{2}, k_{1} k_{2}, d_{1} d_{2}\right)$ code in which codewords form an $n_{1} \times n_{2}$ array such that each row is a codeword from an $\left(n_{1}, k_{1}, d_{1}\right)$ code $C_{1}$, and each column is a codeword from an $\left(n_{2}, k_{2}, d_{2}\right)$ code $C_{2}$, as shown in Fig. 7 [with $n_{i}, k_{i}$, and $d_{i}(i=1,2)$ being the codeword length, dimension, and minimum distance, respectively, of $i$ th component code]. For fiber-optics communications turbo product codes based on BCH component codes are intensively studied, e.g., [4]-[6]. As indicated by Elias [23], turbo product codes can be decoded by sequentially decoding the columns and rows using maximum a posteriori decoding of component codes. However, due to the high complexity of BCJR algorithm [24], low-complexity Chase II algorithm [3], [17] is commonly employed in practical applications [5], [6]. An efficient realization of Chase II algorithm proposed in [18] on an AWGN channel is adopted, however, it has been modified and improved to be applicable in fiber optics channel. Unlike in [3]-[5] and [18], our version of Chase II algorithm does not require scaling and correction factors, and it is briefly outlined below.

1) Determine $p$ least reliable positions starting from (7). Generate $2^{p}$ test patterns to be added to the hard-decision word obtained after (7).

2) Determine the $i$ th $\left(i=1, \ldots, 2^{p}\right)$ perturbed sequence by adding (modulo-2) the test pattern to the hard-decision word (on least reliable positions).

3) Perform the algebraic or hard decoding to create the set of candidate codewords. Simple syndrome decoding is suitable for high-speed transmission and is employed here.
4) Calculate the candidate codeword LLRs using (5).

5) Calculate the extrinsic bit reliabilities for next decoding stage using the (6)-(7).

Extrinsic reliabilities from previous decoding stage are used as inputs processed by next decoding stage, and so on. The iterative procedure is terminated either when a valid codeword is generated or a predetermined number of iterations is reached. In calculating (5) and (6), the following "max-star" operator is applied recursively [19]

$$
\max ^{*}(x, y)=\max (x, y)+\log (1+\exp [-|x-y|])
$$

where the $\max ^{*}$ is defined as $\max ^{*}(x, y)=\log \left(\mathrm{e}^{x}+\mathrm{e}^{y}\right)$. Unlike [3]-[6], because not any approximation is used in calculation of the extrinsic reliabilities, there is no need to introduce the scaling factor $\alpha$ and the correction factor $\beta$.

\section{REFERENCES}

[1] R. H. Morelos-Zaragoza, The Art of Error Correcting Coding. Boston, MA: Wiley, 2002.

[2] O. A. Sab, "FEC techniques in submarine transmission systems," in Proc Optical Fiber Communication Conf., 2001, vol. 2, pp. TuF1-1-TuF1-3.

[3] R. M. Pyndiah, "Near-optimum decoding of product codes: Block turbo codes," IEEE Trans. Commun., vol. 46, no. 8, pp. 1003-1010, Aug. 1998.

[4] O. A. Sab and V. Lemarie, "Block turbo code performances for longhaul DWDM optical transmission systems," in Proc. OFC 2001, vol. 3 , pp. 280-282.

[5] T. Mizuochi et al., "Forward error correction based on block turbo code with 3-bit soft decision for 10-Gb/s optical communication systems," IEEE J. Sel. Topics Quantum Electron., vol. 10, no. 2, pp. 376-386, Mar./Apr. 2004.

[6] - "Next generation FEC for optical transmission systems," in Proc. OFC 2003, vol. 2, pp. 527-528.

[7] I. B. Djordjevic, O. Milenkovic, and B. Vasic, "Generalized low-density parity-check codes for optical communication systems," J. Lightw. Technol., vol. 23, no. 5, pp. 1939-1946, May 2005.

[8] B. Vasic, I. B. Djordjevic, and R. Kostuk, "Low-density parity check codes and iterative decoding for long haul optical communication systems," $J$. Lightw. Technol., vol. 21, no. 2, pp. 438-446, Feb. 2003.

[9] I. B. Djordjevic et al., "Projective plane iteratively decodable block codes for WDM high-speed long-haul transmission systems," J. Lightw. Technol., vol. 22, no. 3, pp. 695-702, Mar. 2004.

[10] O. Milenkovic, I. B. Djordjevic, and B. Vasic, "Block-circulant low-density parity-check codes for optical communication systems," IEEE/LEOS J. Sel. Topics Quantum Electron., vol. 10, no. 2, pp. 294 299, Mar./Apr. 2004.

[11] B. Vasic and I. B. Djordjevic, "Low-density parity check codes for long haul optical communications systems," IEEE Photon. Technol. Lett., vol. 14, no. 8, pp. 1208-1210, Aug. 2002.

[12] S.-Y. Chung et al., "On the design of low-density parity-check codes within $0.0045 \mathrm{~dB}$ of the shannon limit," IEEE Commun. Lett., vol. 5 , no. 2, pp. 58-60, Feb. 2001.

[13] M. C. Davey and D. J. C. MacKay, "Low-density parity check codes over GF(q)," IEEE Commun. Lett., vol. 2, no. 6, pp. 165-167, Jun. 1998.

[14] H. Wymeersch et al., "Log-domain decoding of LDPC codes over GF(q)," in Proc. IEEE Int. Conf. Commun. 2004, vol. 2, pp. 772-776.

[15] I. B. Djordjevic and B. Vasic, "Nonbinary LDPC codes for optical communication systems," IEEE Photon. Technol. Lett., vol. 17, no. 10, pp. 2224 2226, Oct. 2005.

[16] H. Xiao-Yu et al., "Efficient implementations of the sum-product algorithm for decoding of LDPC codes," in Proc. IEEE Global Telecommunications Conf. (Globecom'01) Nov. 25-29, 2001, vol. 2, pp. 1036-1036E.

[17] D. Chase, "A class of algorithms for decoding of block codes with channel measurements information," IEEE Trans. Inf. Theory, vol. IT-18, no. 1, pp. 170-182, Jan. 1972.

[18] S. A. Hirst, B. Honary, and G. Markarian, "Fast Chase algorithm with an application in turbo decoding," IEEE Trans. Commun., vol. 49, no. 10 , pp. 1693-1699, Oct. 2001 
[19] W. E. Ryan, "Concatenated convolutional codes and iterative decoding," in Wiley Encyclopedia in Telecommunications, J. G. Proakis, Ed. New York: Wiley, 2003.

[20] G. Montorsi and S. Benedetto, "Design of fixed-point iterative decoders for concatenated codes with interleavers," IEEE J. Sel. Areas Commun., vol. 19, no. 5, pp. 871-882, May 2001.

[21] B. Vasic, V. Rao, I. B. Djordjevic, R. Kostuk, and I. Gabitov, "Ghost pulse reduction in 40-Gb/s systems using line coding," IEEE Photon. Technol. Lett., vol. 16, no. 7, pp. 1784-1786, Jul. 2004.

[22] I. B. Djordjevic, S. K. Chilappagari, and B. Vasic, "Suppression of intrachannel nonlinear effects using pseudo-ternary constrained codes," $J$. Lightw. Technol, vol. 24, no. 2, pp. 769-774, Feb. 2006.

[23] P. Elias, "Error-free coding," IRE Trans. Inf. Theory, vol. IT-4, pp. 29-37, Sep. 1954

[24] L. R. Bahl, J. Cocke, F. Jelinek, and J. Raviv, "Optimal decoding of linear codes for minimizing symbol error rate," IEEE Trans. Inf. Theory, vol. IT-20, no. 2, pp. 284-287, Mar. 1974.

[25] A. H. Gnauck and P. J. Wintzer, "Optical phase-shift keyed transmission," J. Lightw. Technol., vol. 23, no. 1, pp. 115-130, Jan. 2005.

[26] M. Mansour, "Implementation of LDPC decoders," presented at the IEEE Communication Theory Workshop, Park City, UT, Jun. 13-15, 2005.

[27] T. Richardson, "Error floors of LDPC codes," in Proc. 41 st Allerton Conf. Commun., Control and Comput., Monticello, IL, Oct. 2003, pp. 14261435.

[28] V. Chernyak. (2005, Jan. 10-12). Local theory of BER for LDPC codes: instantons on a tree LANL Workshop on Applications of Statistical Physics to Coding Theory, Santa Fe, New Mexico. [Online]. Available: http://cnls.lanl.gov/ $/$ chertkov/FEC_agenda.htm

[29] R. G. Gallager, Low Density Parity Check Codes. Cambridge, MA: MIT Press, 1963.

[30] S. K. Chilappagari, S. Sankaranarayanan, and B. Vasic, "Error floors of LDPC codes on binary symmetric channel," presented at the IEEE Int. Conf. Commun., (ICC 2006), Istanbul, Turkey, Jun. 11-15, 2006.

[31] B. Vasic, S. K. Chilappagari, and S. Sankaranarayanan, "Error floors of LDPC codes on binary symmetric channel," presented at the IEEE Communication Theory Workshop, Park City, UT, Jun. 13-15, 2005.

[32] P. Humblet and M. Azioglou, "On the bit error rate of lightwave systems with optical amplifiers," J.Lightw. Technol., vol. 9, no. 11, pp. 1576-1582, Nov. 1991.

[33] I. B. Djordjevic and B. Vasic, "Receiver modeling for optically amplified communication systems," Int. J.Electron. Commun. (AEÜ), vol. 57, no. 6, pp. 381-390, 2003.

[34] — "Constrained coding techniques for suppression of intrachannel nonlinear effects in high-speed optical transmission," J. Lightw. Technol., vol. 24, no. 1, pp. 411-419, Jan. 2006.

[35] S. Lin and D. J. Costello, Error Control Coding: Fundamentals and Applications. $\quad$ Englewood Cliffs, NJ: Prentice-Hall, 1983.

[36] D. Declercq and M. P. Fossorier, "Decoding algorithms for LDPC codes over GF(q)," in IEEE Trans. Commun., submitted for publication.

[37] L. Barnault and D. Declercq, "Fast decoding algorithm for LDPC over GF $\left(2^{q}\right)$," in Proc. ITW'03, Paris, France, Mar. 31-Apr. 4, 31-4, 2003 , pp. 70-73.

[38] G. P. Agrawal, Nonlinear Fiber Optics. San Diego, CA: Academic, 2001.

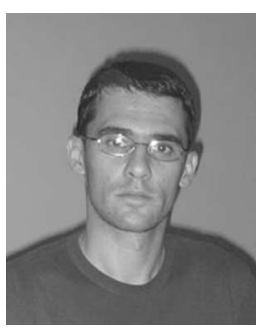

Ivan B. Djordjevic (M'04) received the B.Sc., M.Sc., and Ph.D. degrees from the University of Nis, Nis, Serbia, in 1994, 1997, and 1999, respectively, all in electrical engineering.

He was with the University of the West of England, Bristol, U.K.; University of Bristol, Bristol; Tyco Telecommunications, Eatontown, NJ; National Technical University of Athens, Athens, Greece; State Telecommunications Company (Telecom Serbia), Nis, Serbia; and University of Nis, Nis. Currently, he is an Assistant Research Professor of Electrical and Computer Engineering at the University of Arizona, Tucson. He is the author of more than 100 international publications. His research interests include DWDM fiber-optic communication systems and networks, error control coding, OFDM, CDMA, optical packet switching, free-space optics, communication theory, satellite communications, wireless communications, and coherent communications.

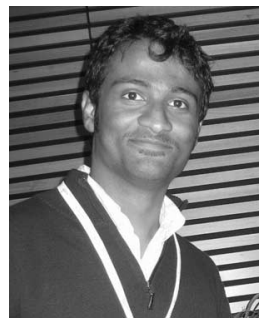

Sundararajan Sankaranarayanan received the B.E. degree in electronics and communications engineering from the University of Madras, Chennai, India, and the M.S. degree in electrical engineering and the Ph.D. degree from the University of Arizona, Tucson. His Ph.D. dissertation was on iterative decoding of linear block codes.

$\mathrm{He}$ is currently with Seagate Technologies, Pittsburgh, PA. In the summers of 2000 and 2002, respectively, he was with the Philips Research, NY, and Seagate Research, PA. In the fall of 2004, he was with the Systems Group of IBM Research Laboratory, Zurich, Switzerland. His research interests include iterative decoders, error floors of LDPC codes, theory of error-control codes, and its applications in magnetic recording systems and optical communication systems.

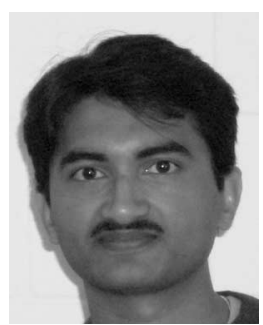

Shashi Kiran Chilappagari received the B.Tech. and M.Tech. degrees from the Indian Institute of Technology, Madras, India, in 2004, both in electrical engineering. He is currently working toward the $\mathrm{Ph} . \mathrm{D}$. degree in digital communications at the University of Arizona, Tucson.

His research interests include error control coding, data compression, and information theory. His specific interests are the analysis of iterative decoding algorithms and investigation of the phenomenon of error floors of LDPC codes.

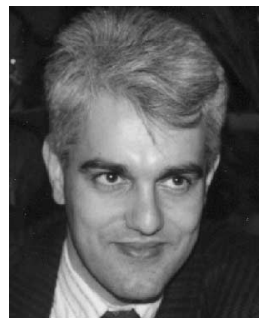

Bane Vasic (S'92-M'93-SM'02) received the B.Sc., M.Sc., and Ph.D. degrees from the University of Nis, Nis, Serbia, in 1990, 1991, and 1994, respectively, all in electrical engineering.

From 1996 to 1997, he was a Visiting Scientist at the Rochester Institute of Technology and Kodak Research, Rochester, NY, where he was involved in research on optical storage channels. During 19982000, he was with Lucent Technologies and Bell Laboratories, Murray Hill, NJ. He was involved in research coding schemes and architectures for highspeed applications and in iterative decoding and low-density parity check codes, as well as development of codes and detectors implemented in Lucent (now Agere) chips. His research interests include coding theory, information theory, communication theory, and digital communications and recording. He is a Faculty Member of the Electrical and Computer Engineering Department, University of Arizona, Tucson.

Dr. Vasic is a Member of the Editorial Board of the IEEE TRANSACTIONS on Magnetics. He served as a Technical Program Chair for the IEEE Communication Theory Workshop, 2003, and as a Coorganizer of the Center for Discrete Mathematics and Theoretical Computer Science Workshops on Opti$\mathrm{cal} /$ Magnetic Recording and Optical Transmission, and Theoretical Advances in Information Recording, 2004. He is also a Coorganizer of the Communication Theory Symposium within the International Conference on Communications (ICC 2006). 